\title{
Population structure of Dover sole Solea solea: RAPD and allozyme data indicate divergence in European stocks
}

\author{
Athanasios Exadactylos ${ }^{1,2, *}$, Audrey J. Geffen ${ }^{1}$, Panagiota Panagiotaki ${ }^{1,2}$, John P. Thorpe ${ }^{1}$ \\ ${ }^{1}$ School of Biological Sciences, University of Liverpool, Port Erin Marine Laboratory, Port Erin, Isle of Man IM9 6JA, British Isles \\ ${ }^{2}$ Present address: Department of Agriculture, Animal Production and Aquatic Environment, School of Agricultural Sciences, \\ University of Thessaly, Fytokou Street, 38446, N. Ionia Magnissias, Greece
}

\begin{abstract}
Randomly amplified polymorphic DNA (PCR-RAPD) analysis was used to estimate genetic variation within and between 6 Northeast Atlantic populations of Dover sole Solea solea (L.). A total of 16 fish were randomly selected from each population, and the results were compared with allozyme variation within and between populations. Results from both methods were in general agreement, but the RAPD technique detected higher levels of genetic variation. Contingency analyses (allele frequencies and hierarchical $F_{\mathrm{ST}}$ and non-hierarchical $F_{\mathrm{DT}}$ ) indicated highly significant genetic heterogeneity between populations. This result is consistent with the life history of Dover sole, which have homing behavior and discrete spawning grounds. Divergence between populations is indicated by cluster analyses of both allozymes and RAPD data. However, allozymes provided a somewhat better fit of data to predictions (higher values of cophenetic correlation of clusters from the goodness-of-fit statistics) and better correlation between genetic and geographical distances (Mantel's r). Both allozyme and RAPD data indicate that the samples can be clustered into two groups; continental Europe (Bay of Biscay and German Bight) and British Isles (Cumbria, Isle of Man, Ireland and North Sea). Despite the small geographical separation at the closest point, the English Channel may provide a barrier to gene flow between populations of Dover sole around the coasts of Britain and those on the coast of continental Europe.
\end{abstract}

KEY WORDS: Allozymes · Gene flow · Genetic structure · Isolation-by-distance • PCR-RAPD . Solea solea

Resale or republication not permitted without written consent of the publisher

\section{INTRODUCTION}

Studies of genetic divergence and population structure in marine species have used various molecular techniques (reviews by e.g. Park \& Moran 1995, Carvalho \& Hauser 1998), the most common being enzyme (allozyme) electrophoresis. This remains the most rapid, simple and inexpensive method for population level studies, where large numbers of samples need to be screened, and it is the technique of choice for analyses of fish stocks (see e.g. Utter et al. 1987, Ward 1989, Utter 1995, Ward \& Grewe 1995, Thorpe et al. 2000). Nevertheless, recent molecular methods may provide higher resolution of genetic structuring within species (Ward \& Grewe 1995). A relatively straightforward method is PCR-RAPD, the random amplification of polymorphic DNA (RAPD) by polymerase chain reaction (PCR) (Welsh \& McClelland 1990, Williams et al. 1990). This provides a simple means of detecting polymorphisms, e.g. for genetic mapping or strain identification (Hadrys et al. 1992).

RAPD has been used in studies on a range of organisms (e.g. nematodes: Caswell-Chen et al. 1992; insects: Black et al. 1992, Chapco et al. 1992), particularly plants (e.g. Huff et al. 1993, Kazan et al. 1993, Peakall et al. 1995, Rossetto et al. 1995) and freshwater 
fish (e.g. Bardakci \& Skibinski 1994, Naish et al. 1995, Williams et al. 1998, Koh et al. 1999, Liu et al. 1999, Nesbo et al. 1999), and in work on endangered species (e.g. frogs: Kimberling et al. 1996; snakes: Gibbs et al. 1994; whales: Martinez \& Pastene 1999; and birds: Haig et al. 1994, Nusser et al. 1996). In marine species its use has been limited (e.g. Allegrucci et al. 1995, Bielawski \& Pumo 1997, Harding et al. 1997, Smith et al. 1997, Todd et al. 1997, Gomes et al. 1998, Heipel et al. 1998, Hirschfeld et al. 1999, Mamouris et al. 1999), and the database on RAPD is not as extensive as that long been available for allozyme studies (see e.g. Thorpe 1982, Nei 1987, Thorpe \& Sole-Cava 1994).

The primary objective of the present study was to compare patterns of allozyme and RAPD variation in 6 Atlantic populations of Dover sole Solea solea (L.), a commercially important soleid flatfish in shallower inshore waters of Europe (Rijnsdorp et al. 1992). Allozyme data on Dover sole were available from an earlier study (Exadactylos et al. 1998; see also Kotoulas et al. 1995, Exadactylos et al. 1999, Exadactylos \& Thorpe 2001) and the intention was to use RAPD to estimate levels of genetic diversity within and between populations based on the same material as used previously by Exadactylos et al. (1998). It was hoped that RAPD might help to differentiate between populations that were very similar in the allozyme study and provide firstly an empirical assessment of the ability of RAPD to resolve interpopulation differences not detected by allozymes, and secondly a direct comparison of levels of divergence revealed by the 2 methods.

PCR-RAPD applies the PCR technique to genomic DNA samples, using randomly constructed oligonucleotides as primers. A single short primer (10 base pairs) and low annealing temperature are combined to obtain specific amplification patterns from individual genomes. These anonymous DNA fragments are separated by size on a standard agarose gel and visualised by ethidium bromide staining. The method can detect extensive polymorphisms and does not require a priori sequence information on the organism studied. Polymorphisms result from either size changes within the amplified region or base changes that alter primer binding (Lynch \& Milligan 1994). Such polymorphisms are usually inherited in a Mendelian fashion and can be used as genetic markers (Bardakci \& Skibinski 1994). PCR-RAPD is simpler, considerably faster and less expensive than other methods for detecting DNA sequence variation (Caetano-Anolles et al. 1991a,b). Since RAPD can be used over numerous loci in the genome, the method is generally attractive for analysis of genetic distance, genetic structure at the population level, breeding applications, and phylogenetic reconstruction (Clark \& Lanigan 1993).
Practical problems in RAPD applications include the difficulty in distinguishing products of different loci with similar molecular weights (co-migration) (see e.g. Grosberg et al. 1996). Polymorphism is detected as band presence versus absence after PCR amplification, since RAPD markers are usually dominant characters (Williams et al. 1990). These phenotypically dominant markers in diploid organisms make it impossible to distinguish between homozygotes and heterozygotes for the dominant allele (Lynch \& Milligan 1994). This problem does not preclude the estimation of allele frequencies necessary for population genetic analysis, but it does reduce the accuracy of such estimates compared to analysis with co-dominant markers, i.e. allozymes (Lynch \& Milligan 1994). Each RAPD locus is treated as a 2 allele system, with the assumption that only 1 of the alleles per locus is amplifiable by the PCR. The existence of multiple amplifiable alleles at a locus is reported to be relatively rare (Clark \& Lanigan 1993), but evidence to the contrary has also been reported (Grosberg et al. 1996). Under random mating the inbreeding index $F_{\mathrm{IS}}$ is zero and the expected genotype frequencies follow Hardy-Weinberg expectations, another assumption to consider in populationgenetic structure analyses with dominant genetic markers (Stewart \& Excoffier 1996).

\section{MATERIALS AND METHODS}

Sample tissue collection and digestion. A total of 96 individuals, 16 from each of the 6 Atlantic populations of Solea solea (3 from the Irish Sea, 2 from the North Sea, 1 from the Bay of Biscay) studied by Exadactylos et al. (1998) were randomly selected for analysis. Allozyme data had shown that Dover sole from the Mediterranean were genetically distinct from those in the Atlantic, so these samples were excluded from the RAPD analysis. The sampling protocol of the 6 Atlantic populations has already been described (Exadactylos et al. 1998). Frozen tissue samples of skeletal muscle stored at $-75^{\circ} \mathrm{C}$ were used for DNA extractions. Preliminary trials were carried out to evaluate DNA extraction techniques. To get good results with any given species, techniques have to be perfected, often by trial and error, and the extraction and PCR methods for Dover sole are given below.

DNA extraction and quality control. Tissue samples were digested in sterile Eppendorf tubes containing a solution of $500 \mathrm{\mu l}$ TNE buffer $(10 \mathrm{mM}$ Tris- $\mathrm{HCl}$, $100 \mathrm{mM} \mathrm{NaCl}, 10 \mathrm{mM}$ EDTA, $\mathrm{pH}$ 8), $50 \mu \mathrm{l}$ Tris- $\mathrm{HCl}$ at $\mathrm{pH} 8,25 \mu \mathrm{l} 25 \%$ SDS and $20 \mu \mathrm{l}$ Proteinase K (Biometra). These were shaken gently and incubated at $55^{\circ} \mathrm{C}$ for $2 \mathrm{~h}$, or until the tissue was dissolved. After tissue digestion, $600 \mu \mathrm{l}$ phenol-chloroform was added 
to the Eppendorf tubes, to dissolve any remains of proteins or enzymes. The tubes were rotated on a mixing machine (Stuart Scientific Rotator Drive STR4, Jencons) at 8.25 speed for $10 \mathrm{~min}$ and then centrifuged (Biofuge 13, Heraeus Sepatech) at $13000 \times g$ for $10 \mathrm{~min}$. The dissolved DNA in the buffer solutions was extracted off the top of the phenol-chloroform layers, using cut pipette tips rather than the normal uncut tips, to avoid breaking of DNA in the sample. The supernatants were added into new Eppendorf tubes containing $600 \mu \mathrm{l}$ chloroform/iso-amyl alcohol. The contents were mixed by rotation for $10 \mathrm{~min}$ and centrifuged for another $10 \mathrm{~min}$, as described above. This step removed phenol-chloroform left in the DNA sample.

The supernatants were removed using cut pipette tips and added to a new set of Eppendorf tubes containing $1 \mathrm{ml}$ of absolute ethanol. Gentle mixing by inverting dissolved any chloroform/iso-amyl impurities left in the sample, $6 \mu \mathrm{l} 3 \mathrm{M}$ sodium acetate was added to the DNA in the ethanol and the tubes were frozen at $-70^{\circ} \mathrm{C}$ for $30 \mathrm{~min}$. This step precipitates the DNA. After thawing, the tubes were centrifuged for $5 \mathrm{~min}$ and excess ethanol was poured off to leave a small white pellet at the bottom of each tube. Pellets were washed with $200 \mu \mathrm{l}$ cold $70 \%$ ethanol and again centrifuged for 5 min. Ethanol was poured off again and the tubes (with pellets at the bottom) were incubated at $\sim 40^{\circ} \mathrm{C}$ for $\sim 20 \mathrm{~min}$ to evaporate excess ethanol. Finally, $50 \mu \mathrm{l}$ of ultrasterile water was added to each pellet. The ultra-sterile water was prepared by deionising, followed by sterilisation with UV radiation to denature enzymes and nucleic acids. DNA samples were stored frozen until analysed.

Most of the samples had good quality DNA, but some yielded little or no DNA, or poor quality, degraded DNA. To assess quality and quantity of DNA, a small aliquot of each sample was run on a $1 \%$ agarose minigel (0.21 g MP agarose; Boehringer-Mannheim) in $30 \mathrm{ml}$ of $1 \times$ Tris-borate-EDTA-buffer (Sigma) pH 8.3, stained with $3 \mu \mathrm{l}$ ethidium bromide (BDH) and assessed under UV light. Samples with clear, sharp, bright bands had good quality DNA, whilst faint bands, or no bands at all had low concentrations or no DNA, respectively. Samples that showed smearing on the top of the gel had badly degraded DNA. Extraction from the original tissue was repeated in these cases.

PCR-RAPD procedure. PCR reactions were performed in $20 \mu \mathrm{l}$ total volume containing $2 \mu \mathrm{l} 10 \times$ reaction buffer (100 mM Tris- $\mathrm{HCl}, \mathrm{pH} 8.8 ; 15 \mathrm{mM} \mathrm{MgCl}_{2 i}$ $500 \mathrm{mM} \mathrm{KCl} ; 1 \%$ Triton X-100; Dynazyme ${ }^{\mathrm{TM}} \mathrm{II}$, Flowgen Instruments), $0.2 \mathrm{mM}(\mathrm{pH} 7)$ of each deoxynucleotide triphosphate (dATP, dCTP, dTTP, dGTP; Amresco), $0.75 \mathrm{mM}$ 10-mer primer (Operon); $1 \mu \mathrm{l}$ genomic DNA and $11.75 \mu \mathrm{l}$ water (molecular biology grade, $\mathrm{BDH})$. The reaction mixture was overlaid with $30 \mu \mathrm{l}$ mineral oil (Sigma). DNA amplification was performed in a thermal cycler (model TCI, Perkin Elmer). The tubes were placed at random in the PCR machine for an initial melting step at $94^{\circ} \mathrm{C}$ for $3 \mathrm{~min}$, before $0.5 \mathrm{U}$ of Taq DNA polymerase (Dynazyne ${ }^{\mathrm{TM}} \mathrm{II}$ ) was added. For the first cycle, denaturation was at $94^{\circ} \mathrm{C}$ for $3 \mathrm{~min}$, annealing at $36^{\circ} \mathrm{C}$ for $30 \mathrm{~s}$, and extension at $72^{\circ} \mathrm{C}$ for $1.5 \mathrm{~min}$. Denaturation time was decreased to $30 \mathrm{~s}$ for the following 43 cycles. A final extension step at $75^{\circ} \mathrm{C}$ was performed for 5 min before the samples were finally cooled off at $4^{\circ} \mathrm{C}$. A negative control with template DNA replaced by water, was performed for each set of amplifications, in order to verify the absence of contamination.

The amplification products were resolved electrophoretically on $1.4 \%$ agarose gels run at $60 \mathrm{~V}$ for 12 to $14 \mathrm{~h}$ in $1 \times$ TBE buffer and fragments were visualised by staining with ethidium bromide (BDH). Sizes were estimated by comparisons with a $1 \mathrm{~kb}$ ladder (Promega) and by running some individuals as reference samples on different gels. Gels were distained for several hours in a bath of distilled water and photographed under UV light with a DS34 Polaroid camera using monochrome negative film (Type 655). In a pilot survey, 25 different 10-base primers (Operon Kits R, $\mathrm{Y}$ and $\mathrm{F}$ ) were evaluated, of which 15 primers gave clear, reproducible, informative marker patterns, coded by 37 polymorphic and 1 monomorphic scorable band fragments (Table 1), and these were selected for the final analysis. Band fragments were 350 to $1150 \mathrm{bp}$ in length and were scored for presence (1) or absence (0),

Table 1. Solea solea. Summary of data obtained on 6 populations by RAPD analysis for 15 primers. Band size: approximate band size range (bp) of polymorphic markers

\begin{tabular}{|llcc|}
\hline Primer codes & $\begin{array}{c}\text { Primer sequence } \\
\left(5^{\prime} \text { to } 3^{\prime}\right)\end{array}$ & $\begin{array}{c}\text { Scorable } \\
\text { bands }\end{array}$ & Band size \\
\hline OPR14 & CAGGATTCCC & 2 & $550-750$ \\
OPR15 & GGACAACGAG & 2 & $350-490$ \\
OPF09 & CCAAGCTTCC & 2 & $590-1150$ \\
OPF07 & CCGATATCCC & 3 & $540-1090$ \\
OPF15 & CCAGTACTCC & 3 & $450-990$ \\
OPR05 & GACCTAGTGG & 2 & $390-580$ \\
OPR16 & CTCTGCGCGT & 3 & $480-730$ \\
OPY15 & AGTCGCCCTT & 4 & $400-830$ \\
OPF17 & AACCCGGGAA & 4 & $590-1020$ \\
OPR10 & CCATTCCCCA & 3 & $350-1030$ \\
OPR11 & GTAGCCGTCT & 3 & $550-840$ \\
OPR13 & GGACGACAAG & 3 & $380-690$ \\
OPR06 & GTCTACGGCA & 2 & $400-640$ \\
OPF03 & CCTGATCACC & 1 & 540 \\
OPR20 & ACGGCAAGGA & 1 & 640 \\
Total & & & \\
15 & & 38 & \\
\hline
\end{tabular}


Table 2. Solea solea. RAPD band frequencies for 38 scorable loci (15 oligonucleotide primers) in 6 Atlantic populations. A and B indicates presence or absence of variable bands. Populations: CUM: Cumbria; IOM: Isle of Man; IRL: Ireland; FRA: Bay of Biscay; GER: German Bight; EAN: East Anglia; North Sea

\begin{tabular}{|c|c|c|c|c|c|c|c|}
\hline \multirow[t]{2}{*}{ Primer code } & \multirow[t]{2}{*}{ Locus } & \multicolumn{6}{|c|}{ Population } \\
\hline & & CUM & IOM & IRL & FRA & GER & EAN \\
\hline \multirow[t]{2}{*}{ OPR14-A } & A & 0.750 & 0.500 & 0.375 & 0.750 & 0.438 & 0.563 \\
\hline & B & 0.250 & 0.500 & 0.625 & 0.250 & 0.563 & 0.438 \\
\hline \multirow[t]{2}{*}{ OPR14-B } & A & 0.438 & 0.219 & 0.563 & 0.281 & 0.375 & 0.656 \\
\hline & B & 0.563 & 0.781 & 0.438 & 0.719 & 0.625 & 0.344 \\
\hline \multirow[t]{2}{*}{ OPR15-A } & $\mathrm{A}$ & 1.000 & 1.000 & 0.750 & 0.563 & 1.000 & 1.000 \\
\hline & B & 0.000 & 0.000 & 0.250 & 0.438 & 0.000 & 0.000 \\
\hline \multirow[t]{2}{*}{ OPR15-B } & $\mathrm{A}$ & 0.438 & 0.438 & 0.250 & 0.500 & 0.281 & 0.250 \\
\hline & B & 0.563 & 0.563 & 0.750 & 0.500 & 0.719 & 0.750 \\
\hline \multirow[t]{2}{*}{ OPF09-A } & A & 1.000 & 1.000 & 0.750 & 0.438 & 0.750 & 0.344 \\
\hline & B & 0.000 & 0.000 & 0.250 & 0.563 & 0.250 & 0.656 \\
\hline \multirow[t]{2}{*}{ OPF09-B } & A & 0.750 & 0.750 & 0.750 & 0.750 & 0.500 & 1.000 \\
\hline & B & 0.250 & 0.250 & 0.250 & 0.250 & 0.500 & 0.000 \\
\hline \multirow[t]{2}{*}{ OPF07-A } & A & 0.750 & 0.750 & 1.000 & 1.000 & 0.750 & 1.000 \\
\hline & B & 0.250 & 0.250 & 0.000 & 0.000 & 0.250 & 0.000 \\
\hline \multirow[t]{2}{*}{ OPF07-B } & $\mathrm{A}$ & 0.750 & 0.563 & 1.000 & 0.281 & 0.656 & 1.000 \\
\hline & B & 0.250 & 0.438 & 0.000 & 0.719 & 0.344 & 0.000 \\
\hline \multirow[t]{2}{*}{ OPF07-C } & A & 0.438 & 0.281 & 0.563 & 0.750 & 0.563 & 1.000 \\
\hline & B & 0.563 & 0.719 & 0.438 & 0.250 & 0.438 & 0.000 \\
\hline \multirow[t]{2}{*}{ OPF15-A } & $\mathrm{A}$ & 1.000 & 1.000 & 1.000 & 1.000 & 1.000 & 0.656 \\
\hline & B & 0.000 & 0.000 & 0.000 & 0.000 & 0.000 & 0.344 \\
\hline \multirow[t]{2}{*}{ OPF15-B } & $\mathrm{A}$ & 1.000 & 1.000 & 1.000 & 1.000 & 1.000 & 1.000 \\
\hline & B & 0.000 & 0.000 & 0.000 & 0.000 & 0.000 & 0.000 \\
\hline \multirow[t]{2}{*}{ OPF15-C } & A & 0.281 & 0.281 & 0.375 & 0.438 & 0.281 & 0.438 \\
\hline & B & 0.719 & 0.719 & 0.625 & 0.563 & 0.719 & 0.563 \\
\hline OPR05-A & A & 0.750 & 0.438 & 0.250 & 0.344 & 0.438 & 1.000 \\
\hline & B & 0.250 & 0.563 & 0.750 & 0.656 & 0.563 & 0.000 \\
\hline OPR05-B & $\mathrm{A}$ & 1.000 & 0.344 & 1.000 & 0.656 & 0.563 & 0.344 \\
\hline & B & 0.000 & 0.656 & 0.000 & 0.344 & 0.438 & 0.656 \\
\hline OPR16-A & A & 0.344 & 0.656 & 0.656 & 0.375 & 0.156 & 0.656 \\
\hline & B & 0.656 & 0.344 & 0.344 & 0.625 & 0.844 & 0.344 \\
\hline OPR16-B & A & 0.750 & 0.563 & 0.563 & 0.438 & 0.281 & 0.375 \\
\hline & B & 0.250 & 0.438 & 0.438 & 0.563 & 0.719 & 0.625 \\
\hline OPR16-C & A & 0.438 & 0.500 & 0.563 & 0.250 & 0.375 & 0.500 \\
\hline & B & 0.563 & 0.500 & 0.438 & 0.750 & 0.625 & 0.500 \\
\hline OPY15-A & A & 0.500 & 0.281 & 0.219 & 0.500 & 0.438 & 0.563 \\
\hline & B & 0.500 & 0.719 & 0.781 & 0.500 & 0.563 & 0.438 \\
\hline OPY15-B & $\mathrm{A}$ & 1.000 & 0.563 & 0.375 & 0.125 & 0.500 & 0.438 \\
\hline & B & 0.000 & 0.438 & 0.625 & 0.875 & 0.500 & 0.563 \\
\hline OPY15-C & A & 0.563 & 0.563 & 0.563 & 0.344 & 0.563 & 0.500 \\
\hline & $\mathrm{B}$ & 0.438 & 0.438 & 0.438 & 0.656 & 0.438 & 0.500 \\
\hline OPY15-D & A & 1.000 & 1.000 & 0.750 & 0.438 & 1.000 & 1.000 \\
\hline & $\mathrm{B}$ & 0.000 & 0.000 & 0.250 & 0.563 & 0.000 & 0.000 \\
\hline OPF17-A & $\mathrm{A}$ & 0.375 & 0.375 & 0.344 & 0.563 & 1.000 & 0.250 \\
\hline & B & 0.625 & 0.625 & 0.656 & 0.438 & 0.000 & 0.750 \\
\hline OPF17-B & $\mathrm{A}$ & 0.156 & 0.250 & 0.438 & 0.750 & 1.000 & 0.250 \\
\hline & B & 0.844 & 0.750 & 0.563 & 0.250 & 0.000 & 0.750 \\
\hline OPF17-C & $\mathrm{A}$ & 0.250 & 0.156 & 0.281 & 1.000 & 1.000 & 0.563 \\
\hline & B & 0.750 & 0.844 & 0.719 & 0.000 & 0.000 & 0.438 \\
\hline OPF17-D & $\mathrm{A}$ & 0.750 & 0.438 & 0.500 & 0.563 & 1.000 & 0.438 \\
\hline & B & 0.250 & 0.563 & 0.500 & 0.438 & 0.000 & 0.563 \\
\hline OPR10-A & $\mathrm{A}$ & 0.656 & 0.438 & 0.125 & 0.344 & 0.563 & 0.344 \\
\hline & $\mathrm{B}$ & 0.344 & 0.563 & 0.875 & 0.656 & 0.438 & 0.656 \\
\hline OPR10-B & $\mathrm{A}$ & 0.375 & 0.250 & 0.219 & 0.563 & 0.438 & 0.344 \\
\hline & $\mathrm{B}$ & 0.625 & 0.750 & 0.781 & 0.438 & 0.563 & 0.656 \\
\hline OPR10-C & A & 0.344 & 0.438 & 0.563 & 0.563 & 0.563 & 0.281 \\
\hline & B & 0.656 & 0.563 & 0.438 & 0.438 & 0.438 & 0.719 \\
\hline OPR11-A & A & 0.656 & 0.656 & 0.656 & 0.563 & 0.750 & 0.438 \\
\hline & B & 0.344 & 0.344 & 0.344 & 0.438 & 0.250 & 0.563 \\
\hline
\end{tabular}


Table 2 (continued)

\begin{tabular}{|c|c|c|c|c|c|c|c|}
\hline \multirow[t]{2}{*}{ Primer code } & \multirow[t]{2}{*}{ Locus } & \multicolumn{6}{|c|}{ Population } \\
\hline & & CUM & IOM & IRL & FRA & GER & EAN \\
\hline \multirow[t]{2}{*}{ OPR11-B } & A & 1.000 & 0.750 & 1.000 & 0.750 & 1.000 & 0.750 \\
\hline & B & 0.000 & 0.250 & 0.000 & 0.250 & 0.000 & 0.250 \\
\hline \multirow[t]{2}{*}{ OPR11-C } & A & 0.344 & 0.281 & 0.250 & 0.281 & 0.281 & 0.344 \\
\hline & B & 0.656 & 0.719 & 0.750 & 0.719 & 0.719 & 0.656 \\
\hline \multirow[t]{2}{*}{ OPR13-A } & A & 0.656 & 0.656 & 0.344 & 1.000 & 0.563 & 1.000 \\
\hline & B & 0.344 & 0.344 & 0.656 & 0.000 & 0.438 & 0.000 \\
\hline \multirow[t]{2}{*}{ OPR13-B } & A & 1.000 & 0.438 & 0.063 & 0.656 & 0.656 & 0.156 \\
\hline & B & 0.000 & 0.563 & 0.938 & 0.344 & 0.344 & 0.844 \\
\hline \multirow[t]{2}{*}{ OPR13-C } & A & 0.500 & 0.656 & 1.000 & 0.656 & 1.000 & 1.000 \\
\hline & B & 0.500 & 0.344 & 0.000 & 0.344 & 0.000 & 0.000 \\
\hline \multirow[t]{2}{*}{ OPR06-A } & A & 0.250 & 0.563 & 0.219 & 0.250 & 0.250 & 0.281 \\
\hline & B & 0.750 & 0.438 & 0.781 & 0.750 & 0.750 & 0.719 \\
\hline \multirow[t]{2}{*}{ OPR06-B } & A & 0.750 & 1.000 & 1.000 & 0.750 & 0.375 & 1.000 \\
\hline & B & 0.250 & 0.000 & 0.000 & 0.250 & 0.625 & 0.000 \\
\hline \multirow[t]{2}{*}{ OPF03-A } & A & 1.000 & 0.656 & 1.000 & 0.656 & 1.000 & 1.000 \\
\hline & B & 0.000 & 0.344 & 0.000 & 0.344 & 0.000 & 0.000 \\
\hline \multirow[t]{2}{*}{ OPR20-A } & A & 1.000 & 0.438 & 0.375 & 0.563 & 1.000 & 1.000 \\
\hline & B & 0.000 & 0.563 & 0.625 & 0.438 & 0.000 & 0.000 \\
\hline
\end{tabular}

generating a matrix of RAPD phenotypes (for further details see Exadactylos 1997).

Data analysis. The following assumptions were made about RAPD profiles in Dover sole: (1) all RAPD loci showed complete dominance, and the absence of a band indicated the genotype of a homozygote for the recessive allele $\left(q^{2}\right),(2)$ all loci had 2 alleles with frequencies $p$ and $q$, and only 1 allele amplified to produce a band; polymorphic bands segregated in a Mendelian fashion, (3) all populations were in HardyWeinberg equilibrium $\left(p^{2}+2 p q+q^{2} ; p+q=1\right)$ (Lynch \& Milligan 1994) and the calculated genetic diversity represents that expected under Hardy-Weinberg equilibrium. Despite the possibility of underestimating (heterozygotes are undetected in visible dominant markers) or overestimating (band frequencies are below $50 \%$ ) genetic diversity, RAPD provides useful estimates of genetic variation allowing for the necessary assumptions (Kimberling et al. 1996).

The entire data set from 96 individuals was used for the analysis, including the single monomorphic marker. The computer programme RapdBios (Black 1993) provides input files suitable for Biosys-2 (Swofford \& Selander 1989), which was used to calculate allele frequencies, heterozygosity estimates and standard genetic analyses of population structure. Heterogeneity $\chi^{2}$ analyses of allele frequencies between populations and groupings were performed by hierarchical analysis using Biosys-2. The sequential Bonferroni procedure was employed to control the probability of incorrectly rejecting null hypotheses at the 0.005 significance level (Holm 1979).

Calculation of Wright's (1978) non-hierarchical $F_{\mathrm{DT}}$ was carried out in Biosys-2 to facilitate comparison of the RAPD data set with that obtained from the allozyme study (Exadactylos et al. 1998). Weir \& Cockerham's (1984) unbiased estimates of $F$ statistics were calculated using the $\operatorname{Rapd} F_{\mathrm{ST}}$ programme, version 3.0 (Black 1995), using the weighted mean frequency of alleles and their deviation from zero (significant population differentiation) and were tested with a contingency $\chi^{2}$ table: $\chi^{2}=2 N F_{\mathrm{ST}} ;$ df $=k-1$, where $N$ is the total number of individuals sampled across $k$ populations. Contemporary estimates of gene flow $\left(N_{\mathrm{e}} m\right)$ were calculated using Slatkin's (1993) approximation, assuming equilibrium between migration and genetic drift.

Two methods of cluster analyses were performed, UPGMA using Modified Rogers Distance $\left(D_{\mathrm{T}}\right)$ and Wagner procedure using Prevosti's Distance (Wright 1978), with the fit of the dendrograms assessed by goodness of fit statistics. The relation between matrices of genetic and linear geographic distances was examined with Mantel's (1967) test of time-spacing clustering using the Ntsys programme (Rohlf 1993). Resulting $r$ values are interpreted as correlation coefficients; 1 : positive correlation, -1 : negative correlation. UPGMA and Wagner procedure dendrograms, Mantel's test, $N_{\mathrm{e}} m, F_{\mathrm{ST}}$ and $F_{\mathrm{DT}}$ were calculated and compared for both data sets (allozymes and RAPD) on the 6 Atlantic populations of Solea solea.

\section{RESULTS}

We used 8 experimental protocols to establish whether the amplification products originated from Solea solea DNA or were artifacts of the amplification 
Table 3. Solea solea. Genetic variability at 38 loci in 6 populations of A locus is considered polymorphic if the frequency of the most common allele does not exceed 0.99. Sample size: sample size per locus; alleles: number of alleles per locus (mean and SE); polymorphism: percentage of polymorphic loci; $\mathrm{H}$ : heterozygosity, expected unbiased estimate (Nei 1978) under Hardy-Weinberg equilibrium (mean and SE)

\begin{tabular}{|lcccc|}
\hline Population & $\begin{array}{c}\text { Sample } \\
\text { size }\end{array}$ & Alleles & $\begin{array}{c}\text { Poly- } \\
\text { morphism }\end{array}$ & $H$ \\
\hline 1. CUM & 16 & $1.7(0.1)$ & 100 & $0.313(0.034)$ \\
2. IOM & 16 & $1.8(0.1)$ & 100 & $0.385(0.029)$ \\
3. GER & 16 & $1.7(0.1)$ & 100 & $0.315(0.036)$ \\
4. IRL & 16 & $1.8(0.1)$ & 100 & $0.326(0.033)$ \\
5. EAN & 16 & $1.7(0.1)$ & 100 & $0.301(0.037)$ \\
6. FRA & 16 & $1.9(0.1)$ & 100 & $0.393(0.027)$ \\
Total & & & & $0.339(0.033)$ \\
& & & & \\
\hline
\end{tabular}

procedure; artifacts are a known problem in RAPD amplifications if parameters are not precisely regulated (see e.g. Ellsworth et al. 1993). Trial runs were carried out to test the effect of $\mathrm{Mg}^{++}$, DNA, dNTP and Taq polymerase concentrations, which can affect the size and the number of amplified products. Consistent, reproducible results were obtained by standardising the reaction conditions to those given above (see also Exadactylos 1997). Estimated allele frequencies at RAPD-

Table 4. Solea solea. Contingency $\chi^{2}$ analysis for heterogeneity of allele frequencies at all loci for six populations. Two alleles were found in each locus. ${ }^{* *} \mathrm{p}<10^{-4}{ }^{* * *} \mathrm{p}<10^{-6}$; overall $\chi^{2}=1386.9, \mathrm{df}=185, \mathrm{p}=10^{-10}$

\begin{tabular}{|ll|}
\hline Locus & $\chi^{2}, \mathrm{df}=5$ \\
\hline OPR15-A & $55.24^{* * *}$ \\
OPF09-A & $59.45^{* * *}$ \\
OPF07-A & $27.43^{* *}$ \\
OPF07-B & $58.59^{* * *}$ \\
OPF07-C & $41.74^{* * *}$ \\
OPF15-A & $58.34^{* * *}$ \\
OPR05-A & $51.38^{* * *}$ \\
OPR05-B & $62.01^{* * *}$ \\
OPR16-A & $29.18^{* *}$ \\
OPY15-B & $53.00^{* * *}$ \\
OPY15-D & $73.49^{* * *}$ \\
OPF17-A & $47.48^{* * *}$ \\
OPF17-B & $71.298^{* * *}$ \\
OPF17-C & $93.06^{* * *}$ \\
OPF17-D & $33.16^{* * *}$ \\
OPR11-B & $27.43^{* *}$ \\
OPR13-A & $50.53^{* * *}$ \\
OPR13-B & $78.36^{* * *}$ \\
OPR13-C & $50.66^{* * *}$ \\
OPR06-B & $64.00^{* * *}$ \\
OPF03-A & $49.69^{* * *}$ \\
OPR20-A & $74.27^{* * *}$ \\
\hline
\end{tabular}

encoding loci varied between populations (Table 2). From a total of 38 loci, 37 were $100 \%$ polymorphic in all 6 populations. Table 3 shows genetic diversity estimates for all 38 markers over all samples. The mean expected genetic heterozygosity estimate was very high $(H=0.339 \pm 0.033)$ but varied little between populations. Heterozygosity was not significantly related to latitude $\left(r=0.616, F_{1,5}=2.448, \mathrm{p}=0.193\right)$.

Contingency $\chi^{2}$ analysis for heterogeneity of allele frequencies between populations (Table 4) indicated significant differences at 22 loci even after the Bonferroni correction (Holm 1979, Rice 1989) at $a^{\prime}=0.005$ for multiple tests. The samples were grouped at several spatial scales, but significant differences in allele frequencies within a grouped sample were detected only within the 4 British (Cumbria, Isle of Man, Ireland and North Sea) and the 2 European coast (Bay of Biscay and German Bight) populations (Table 5). Overall, there was extensive heterogeneity between populations, even greater than that observed with the allozyme data set (Exadactylos et al. 1998). The most marked variation was a significant northwest to southeast pattern of population differentiation, as also indicated by the allozyme allele frequencies.

Population differentiation (mean unbiased $F_{\mathrm{ST}}$ ) estimated from Rapd $F_{\mathrm{ST}}$ was 0.193 and was statistically significant $\left(\chi^{2}=37.056\right.$; df $\left.=5, \mathrm{p}<10^{-6}\right)$. Weir \& Cockerham's $F_{\mathrm{ST}}$ estimates were significantly greater than zero for 21 loci (Table 6), indicating a high level of population differentiation. The locus with the greatest overall genetic variation was OPF17-C; the A allele had the greatest $F_{\mathrm{ST}}$ value and was therefore the most variable between the sampling locations. Its frequency increased gradually from northwest (Irish Sea) to southeast (European coast) (Fig. 1). The mean estimate of Wright's non hierarchical $F_{\mathrm{DT}}$ calculated from Biosys- 2 was 0.162 and was also significant $\left(\chi^{2}=31.104 ; \mathrm{df}=5, \mathrm{p}<0.001\right)$.

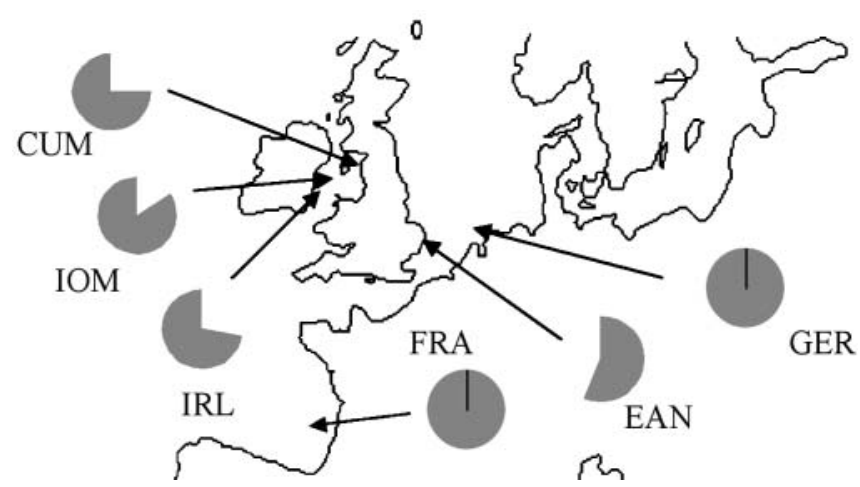

Fig. 1. Solea solea. Variation of allele frequencies at the locus OPF17-C in 6 populations. Shaded part of the circle represents the A allele with the greatest $F_{\mathrm{ST}}$ value, i.e. the most variable. Populations: see Table 2 
Table 5. Solea solea. Significant probability values from contingency $\chi^{2}$ analyses for independence of allele frequencies within samples from the Irish Sea and from the European Coast. -: not significant

\begin{tabular}{|ccc|}
\hline Locus & Irish Sea & European Coast \\
\hline OPR14-B & - & $<4 \times 10^{-4}$ \\
OPR15-A & $<2 \times 10^{-5}$ & - \\
OPF09-A & - & $<1 \times 10^{-6}$ \\
OPF07-A & $<3 \times 10^{-4}$ & $<4 \times 10^{-5}$ \\
OPF07-B & $<3 \times 10^{-4}$ & $<1 \times 10^{-6}$ \\
OPF07-C & - & $<1 \times 10^{-6}$ \\
OPF15-A & - & $<1 \times 10^{-6}$ \\
OPR05-A & - & $<1 \times 10^{-6}$ \\
OPR05-B & - & $<1 \times 10^{-6}$ \\
OPY15-B & $<2 \times 10^{-4}$ & $<1 \times 10^{-6}$ \\
OPY15-D & $<1 \times 10^{-6}$ & $<1 \times 10^{-5}$ \\
OPF17-A & $<2 \times 10^{-5}$ & - \\
OPF17-B & $<3 \times 10^{-4}$ & - \\
OPF17-C & - & $<4 \times 10^{-4}$ \\
OPF17-D & $<2 \times 10^{-5}$ & - \\
OPR10-A & - & $<3 \times 10^{-4}$ \\
OPR11-B & $<3 \times 10^{-4}$ & $<4 \times 10^{-5}$ \\
OPR13-A & $<2 \times 10^{-6}$ & $<1 \times 10^{-6}$ \\
OPR13-B & - & $<1 \times 10^{-6}$ \\
OPR13-C & $<3 \times 10^{-5}$ & $<1 \times 10^{-6}$ \\
OPR06-B & $<3 \times 10^{-4}$ & $<1 \times 10^{-6}$ \\
OPF03-A & $<3 \times 10^{-5}$ & $<1 \times 10^{-6}$ \\
OPR20-A & $<2 \times 10^{-6}$ & $<1 \times 10^{-6}$ \\
& & \\
\hline & & \\
\hline & &
\end{tabular}

Gene flow estimates of approximately one effective migrant per generation $\left(N_{\mathrm{e}} m\right)$ were calculated from these $F_{\mathrm{ST}}$ values. This was considerably lower than the migration rate previously estimated from the allozyme data (approximately 10 migrants per generation) (Table 7). If populations are currently at an equilibrium between migration and genetic drift, an estimate of $N_{\mathrm{e}} m=1$ is sufficiently low to imply population differentiation, as is also indicated from the contingency $\chi^{2}$ analyses (Table 4 ) by the significant allele frequency differences between populations.

UPGMA (Sneath \& Sokal 1973) and Wagner procedure (Wright 1978) clustering of Modified Rogers and Prevosti's genetic distances respectively, further illustrated population relationships (Figs. $2 \& 3$ ). The
Table 6. Solea solea. Significant Weir \& Cockerham's (1984) $\mathrm{F}_{S T}$ values and gene flow estimates, number of effective migrants $\left(\mathrm{N}_{\mathrm{e}} \mathrm{m}\right)$ for 6 populations. All are significant at $\mathrm{p}<10^{-5}$

\begin{tabular}{|lcc|}
\hline Locus & $F_{\mathrm{ST}}$ & $N_{\mathrm{e}} m$ \\
\hline OPR15-A & 0.285 & 0.6 \\
OPF09-A & 0.311 & 0.6 \\
OPF07-A & 0.143 & 1.5 \\
OPF07-B & 0.298 & 0.6 \\
OPF07-C & 0.212 & 0.9 \\
OPF15-A & 0.313 & 0.5 \\
OPR05-A & 0.268 & 0.7 \\
OPR05-B & 0.326 & 0.5 \\
OPY15-B & 0.270 & 0.7 \\
OPY15-D & 0.380 & 0.4 \\
OPF17-A & 0.245 & 0.8 \\
OPF17-B & 0.365 & 0.4 \\
OPF17-C & 0.474 & 0.3 \\
OPF17-D & 0.171 & 1.2 \\
OPR11-B & 0.143 & 1.5 \\
OPR13-A & 0.265 & 0.7 \\
OPR13-B & 0.396 & 0.4 \\
OPR13-C & 0.267 & 0.7 \\
OPR06-B & 0.324 & 0.5 \\
OPF03-A & 0.267 & 0.7 \\
OPR20-A & 0.379 & 0.4 \\
Total & 0.193 & 1.0 \\
OP & & \\
\hline
\end{tabular}

Wagner procedure tree gave a better fit according to the goodness of fit statistics. The observed cophenetic correlation was 0.887, and the allozyme cophenetic correlation was 0.96, suggesting a better agreement between the original matrix and the phenogram than with the RAPD data. The matrix of Modified Rogers Distance $\left(D_{\mathrm{T}}\right)$ and the pairwise estimates of linear geographic distances are shown in Table $8 . D_{\mathrm{T}}$ ranged from 0.232 between the Isle of Man and eastern Irish coast populations to 0.342 between the 2 North Sea populations, indicating little genetic divergence between these populations. Both dendrograms produced a cluster of the geographically proximate Irish Sea populations, which were related to the East Anglian coast population. The Bay of Biscay population was closely related to the German Bight population, as indicated by the allozyme data as well.

Table 7. Solea solea. Estimates of population differentiation (F-statistics), gene flow and correlation of genetic distance with approximate linear geographic distance for PCR-RAPD and allozyme data sets. Population: number of populations studied; $N$ : number of fish screened; $F_{\mathrm{ST}}$ : Weir \& Cockerham (1984); $N_{\mathrm{e}} m(1)$ : gene flow calculated from Weir \& Cockerham's $F_{\mathrm{ST}}$ using $\operatorname{Rapd} F_{\mathrm{ST}}\left(\right.$ Black 1993); $F_{\mathrm{DT}}$ : Wright (1978); $N_{\mathrm{e}} m$ (2): gene flow calculated from Wright's (1978) $F_{\mathrm{DT}}$ using Biosys-2; r: correlation coefficient; Mantel's p: Mantel's probability using 1000 permutations

\begin{tabular}{|lcccccccc|}
\hline Method & Population & $N$ & $F_{\mathrm{ST}}$ & $N_{\mathrm{e}} m(1)$ & $F_{\mathrm{DT}}$ & $N_{\mathrm{e}} m(2)$ & $\mathrm{r}$ & Mantel's p \\
\hline PCR-RAPD & 6 & 96 & 0.193 & 1.045 & 0.162 & 1.293 & 0.37116 & 0.917 \\
Allozymes & 7 & 303 & 0.021 & 11.655 & 0.0262 & 9.292 & 0.54204 & 0.976 \\
\end{tabular}




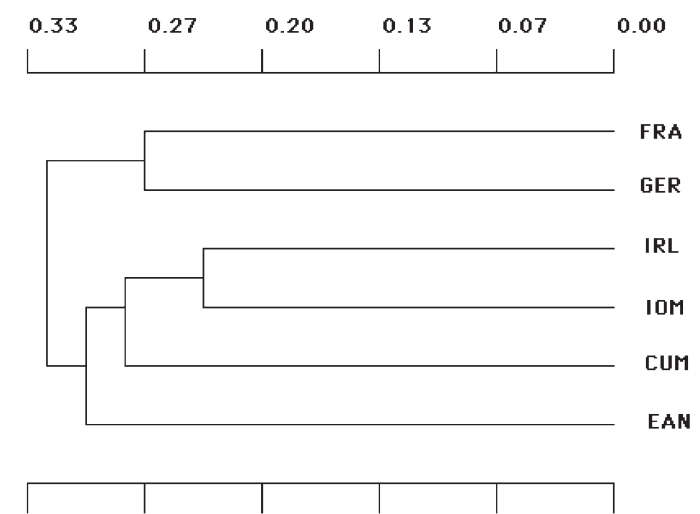

Fig. 2. Solea solea. UPGMA dendrogram of 6 populations, using Modified Rogers Distance $\left(D_{\mathrm{T}}\right)$ (Wright 1978). Goodness of fit statistics: Farris (1972) $f=0.211$; Prager \& Wilson (1976) $F=4.701 ; \%$ SD (Fitch \& Margoliash 1967) $=6.174$; cophenetic correlation $=0.819$. Populations: see Table 2

Mantel's test shows that genetic distance increased with geographical distance $(r=0.37 ; t=1.385)$, but the correlation coefficient was not statistically significant (Table 7). Correlation of geographic distance with genetic distance was also positive for the allozyme data set yielding higher $r$ and $t$ values $(r=0.54$; $t=1.978)$, but also statistically non-significant.

\section{DISCUSSION}

\section{Comparison of RAPD and allozyme data}

The fish used for this study had been previously investigated for allozyme variation (Exadactylos et al. 1998), allowing a direct comparison of the results of the 2 techniques. However, the assumptions involved are quite different. Allozyme markers are usually codominant and the genetic basis of their variation is well understood (Allegrucci et al. 1995), although the method is less sensitive in detecting genetic variation than RAPD (e.g. Grosberg et al. 1996). On the other

Table 8. Solea solea. Estimates of Modified Rogers distance $\left(D_{\mathrm{T}}\right)$ (below the diagonal) and linear coastal geographic distances between headlands in $\mathrm{km}$ (above the diagonal) for 6 Atlantic populations. Abbreviations: see Fig. 1

\begin{tabular}{|lcccccc|}
\hline Population & FRA & IRL & IOM & EAN & GER & CUM \\
\hline FRA & & 1012.5 & 1125.0 & 1375.0 & 1725.0 & 1200.0 \\
IRL & 0.308 & & 112.5 & 1162.5 & 1512.5 & 187.5 \\
IOM & 0.295 & 0.232 & & 1275.0 & 1625.0 & 75.0 \\
EAN & 0.314 & 0.285 & 0.290 & & 350.0 & 1350.0 \\
GER & 0.269 & 0.325 & 0.320 & 0.342 & \multirow{2}{*}{0.299} & 1700.0 \\
CUM & 0.336 & 0.307 & 0.244 & 0.318 & & \\
\hline
\end{tabular}

hand, RAPD markers are usually dominant, and more loci need to be screened to obtain the same accuracy of estimates of genetic variation levels (Lynch \& Milligan 1994).

The 27 polymorphic allozyme loci studied by Exadactylos et al. (1998) provide a comparison with variation at 37 polymorphic RAPD markers. Allozyme and RAPD markers produced similar results, but RAPD markers detected more variation in the Dover sole populations studied by Exadactylos et al. (1998) and Kotoulas et al. (1995). There are no direct comparisons of allozymes with RAPD in Dover sole in the literature, but higher levels of variation have been observed with RAPD as compared to allozymes in fish (e.g. Allegrucci et al. 1995, Naish et al. 1995), grasses (e.g. Peakall et al. 1995), birds (e.g. Haig et al. 1994), insects (e.g. Puterka et al. 1993) and bacteria (e.g. Wang et al. 1993). RAPD probably detects more variation within or between populations due to the number of markers available, which is effectively unlimited, in contrast to the limited number of allozyme markers (Clark \& Lanigan 1993).

Percentage of polymorphism and genetic diversity estimates were very high, but because of dominance, RAPD cannot provide totally reliable estimates of heterozygosity (Welsh \& McClelland 1990, 1991), nor direct interpretation of allele frequencies (Heun \& Helentjaris 1993), without making several assumptions (see above). Nevertheless, there was no clear general overall geographic trend to either RAPD or allozyme allele frequencies over the range of polymorphic loci studied. 
The $\chi^{2}$ contingency analyses (allele frequencies and hierarchical $F_{\mathrm{ST}}$ and non- hierarchical $F_{\mathrm{DT}}$ ) indicated a highly significant heterogeneity between populations and between groupings (northwest versus southeast populations) in the results from both techniques. This is consistent with the life history of Dover sole, its homing behavior (e.g. Rijnsdorp et al. 1992) and discrete spawning areas (e.g. Symonds \& Rogers 1995). These characteristics serve to reduce the homogenisation of population allele frequencies between spawning stocks. Another reason may be the absence further north of some alleles present in the southern European populations. The progressive decline in allozyme allelic diversity northwards is a possible consequence of a population bottleneck, founder effect, genetic drift, or a combination of these (Exadactylos et al. 1998), particularly since the populations in southern Europe were more genetically variable than those from northern Europe; similar mechanisms have been proposed to explain the general tendency for populations to exhibit reduced genetic variability in areas glaciated during the Pleistocene (e.g. Highton \& Webster 1976, Bellemin et al. 1978, Vaisanen \& Lehvasliho 1984, Singh \& Rhomberg 1987). The RAPD data do not support the 'bottleneck' hypothesis of Exadactylos et al. (1998), since levels of genetic variation are similar within all populations sampled and the small differences that do exist show no obvious geographic trend.

The disjunction in the RAPD data between Irish Sea and European coast populations indicates reduced exchange of migrants between these regions, confirming the earlier allozyme study. Apparent divergence between these regions is shown by the cluster analyses (UPGMA, Wagner procedure) of both allozyme and RAPD data. However, allozymes provided a slightly better fit of data to genetic distance predictions (higher values of cophenetic correlation of clusters from the goodness of fit statistics) and better correlation between genetic and geographic distances (Mantel's r); this may be due the small sample sizes for RAPD. For such studies allozymes remain valuable for many types of population study despite the development of new genetic markers (Schaal et al. 1991, Ward \& Grewe 1995, Thorpe et al. 2000). Both allozyme and RAPD cluster analyses show a strong similarity between the Bay of Biscay and the German Bight populations, and suggest a similarity between the three Irish Sea and the East Anglian populations. The possible movement of migrants through the English Channel, previously suggested by Exadactylos et al. (1998) from allozyme data, was also supported by the RAPD results and by earlier tagging experiments (Greer-WaIker \& Emerson 1990) indicating a seasonal migration of Dover sole through the Straits of Dover.
Thus both RAPD and allozyme data indicate a degree of genetic separation between Solea solea populations from the coasts of the British Isles and the coast of continental Europe. This is surprising given the short closest distance $(<40 \mathrm{~km})$ between the coasts of England and France (at the Straits of Dover). However, the depth of the Straits of Dover (50 to $100 \mathrm{~m}$ ) may inhibit Dover sole from crossing the Channel, as they favor shallow coastal waters (Rijnsdorp et al. 1992) and are rarely found below $70 \mathrm{~m}$ (Wheeler 1969). Deeper water may constitute a barrier to gene flow. For example, Allcock et al. (1997) concluded that genetic differentiation between populations of the Antarctic octopus Pareledone turqueti resulted from depths which the species was unable to cross. Other factors, for example unsuitable substratum or strong tidal currents, could also be responsible.

\section{DNA variation between populations}

The apparent absence of isolation-by-distance (nonsignificant correlation of genetic with geographic distance) in both the allozyme and RAPD studies suggests that Solea solea is not at genetic equilibrium (Slatkin 1993), in which case the $F$ statistics will underestimate the degree of differentiation and overestimate gene flow (Slatkin 1985). Estimated $N_{\mathrm{e}} m$ values of approximately 1 migrant into the average deme per generation theoretically indicate sufficient gene flow between populations to prevent population differentiation, if populations are currently at an equilibrium between migration and genetic drift.

Most of the populations sampled came from areas that were not immersed until less than $10000 \mathrm{yr}$ BP, after the end of the last ice age (McCave et al. 1977), i.e. very recently in evolutionary terms (Lauder \& Liem 1983, Exadactylos \& Thorpe 2001), so that it is likely that genetic equilibrium has not been reached and thus, our estimates of gene flow are probably high.

$F_{\mathrm{ST}} / F_{\mathrm{DT}}$ results may be inaccurate also because of unreliable estimates of within population variation derived from limited sample sizes. Gene flow values from such $F_{\mathrm{ST}}$ estimates should thus be interpreted with caution, although they are a useful relative measure of variation between populations (Larcson et al. 1989). Overall, on the basis of the RAPD loci examined, the population differentiation observed in the Atlantic is primarily the result of random genetic drift or differential selection. Alternative means of detecting polymorphism in Solea solea, including the use of singlelocus minisatellite probes and mtDNA analysis, may provide further clarification. 
Acknowledgements. We thank A. D. Rijnsdorp and his colleagues at RIVO-DLO, F. Lagardere, J. P. Lagardere and crew at La Rochelle, France, B. Harley, D. J. Symonds, and B. Turner from the CEFAS laboratory at Lowestoft, England, P. Newton and his colleagues at DANI, Belfast and the crew of RV 'Roagan' for providing us with the samples. We also thank J. D. D. Bishop, D. Heipel, R. D. M. Nash and A. Troumbis for advice and comments on the work. A. Exadactylos acknowledges the financial support of the Greek Scholarship Foundation

\section{LITERATURE CITED}

Allcock AL, Brierley AS, Thorpe JP, Rodhouse PG (1997) Restricted gene flow and evolutionary divergence between geographically separated populations of the Antarctic octopus Pareledone turqueti. Mar Biol 129:97-102

Allegrucci G, Caccone A, Cataudella S, Powell JR, Sbordoni V (1995) Acclimation of the European sea bass to freshwater: monitoring genetic changes by RAPD polymerase chain reaction to detect DNA polymorphisms. Mar Biol 121:591-599

Bardakci F, Skibinski DOF (1994) Application of the RAPD technique in tilapia fish: species and subspecies identification. Heredity 73:117-123

Bellemin J, Adest G, Gorman GC (1978) Genetic uniformity in northern populations of Thamnopsis sirtalis (Serpentes: Colubridae). Copeia 1978:150-151

Bielawski JP, Pumo DE (1997) Randomly amplified polymorphic DNA (RAPD) analysis of Atlantic Coast striped bass. Heredity 78:32-40

Black B (1993) RAPDBIOS - A FORTRAN program to convert PCR-RAPD files to BIOSYS (DATYPE=3) files. Colorado State University, Fort Collins, Colorado

Black B (1995) RAPDF $F_{\mathrm{ST}} 3.0-\mathrm{A}$ FORTRAN program to estimate $F_{\mathrm{ST}}$ and effective migration rates among subpopulations using PCR-RAPD files. Colorado State University, Fort Collins, CO

Black WC, Du Teau NM, Puterka GJ, Nechols JR, Pettorini JM (1992) Use of the RAPD polymerase chain reaction to detect DNA polymorphisms in aphids (Homoptera: Aphididae). Bull Ent Res 82:151-159

Caetano-Anolles G, Bassam BJ, Gresshoff PM (1991a) DNA amplification fingerprinting using very short arbitrary oligonucleotide primers. Biotechnology 9:553-556

Caetano-Anolles G, Bassam BJ, Gresshoff PM (1991b) DNA amplification fingerprinting: a strategy for genome analysis. Plant Mol Biol Rep 9:294-307

Carvalho GR, Hauser L (1998) Advances in the molecular analysis of fish population structure. Ital J Zool 65:21-33

Caswell-Chen EP, Williamson VM, Wu FF (1992) RAPD analysis of Heterodera cruciferae and $H$. schachtii populations. J Nematol 24:343-351

Chapco W, Ashton NW, Martel RKB, Antonishyn N (1992) A feasibility study of the use of random amplified polymorphic DNA in the population genetics and systematics of grasshoppers. Genome 35:569-574

Clark AG, Lanigan CMS (1993) Prospects for estimating nucleotide divergence with RAPDs. J Mol Biol Evol 10: 1096-1111

Ellsworth DL, Rittenhouse KD, Honeycutt RL (1993) Artifactual variation in randomly amplified polymorphic DNA (RAPD) banding patterns. Bio Techn 14:214-217

Exadactylos A (1997) Population genetics of the Dover sole, Solea solea (Linnaeus, 1758) (Teleostei: Soleidae). PhD thesis, University of Liverpool
Exadactylos A, Thorpe JP (2001) Allozyme variation and genetic inter-relationships between seven flatfish species (Pleuronectiformes). Zool J Linn Soc 132:487-499

Exadactylos A, Geffen AJ, Thorpe JP (1998) Population structure of the Dover sole, Solea solea (L.) in a background of high gene flow. J Sea Res 40:117-129

Exadactylos A, Geffen AJ, Thorpe JP (1999) Growth and genetic variation in hatchery-reared larval and juvenile Dover sole, Solea solea (L.). Aquaculture 176:209-226

Farris JS (1972) Estimating phylogenetic trees from distance matrices. Am Nat 106:645-668

Fitch WM, Margoliash E (1967) Construction of phylogenetic trees. Science 155:279-284

Gibbs HL, Prior KA, Weatherhead PJ (1994) Genetic analysis of populations of threatened snake species using RAPD markers. Mol Ecol 3:329-337

Gomes C, Dales RBG, Oxenford HA (1998) The application of RAPD markers in stock discrimination of the four-wing flying fish, Hirundichthys affinis in the central western Atlantic. Mol Ecol 7:1029-1039

Greer-Walker M, Emerson L (1990) The seasonal migration of soles (Solea solea) through the Dover strait. Neth J Sea Res 25:417-422

Grosberg RK, Levitan DR, Cameron BB (1996) Characterization of genetic structure and genealogies using PCRRAPD markers: A random primer for the novice and nervous. In: Ferraris JD, Palumbi SR (eds) Molecular zoology: advances, strategies and protocols. Wiley-Liss, New York, p 67-100

Hadrys H, Balick M, Scierwater B (1992) Applications of random amplified polymorphic DNA (RAPD) in molecular ecology. Mol Ecol 1:55-63

Haig SM, Rhymer JM, Heckel DG (1994) Population differentiation in randomly amplified polymorphic DNA of redcockaded woodpeckers, Picoides borealis. Mol Ecol 3: 581-595

Harding GC, Kenchington EL, Bird CJ, Pezzack DS, Landry DC (1997) Genetic relationships among subpopulations of the American lobster (Homarus americanus) as revealed by random amplified polymorphic DNA. Can J Fish Aquat Sci 54:1762-1771

Heipel DA, Bishop JDD, Brand AR, Thorpe JP (1998) Population genetic differentiation of the great scallop Pecten maximus in western Britain investigated by randomly amplified polymorphic DNA. Mar Ecol Prog Ser 162: 163-171

Heun M, Helentjaris T (1993) Inheritance of RAPDs in $F_{1}$ hybrids of corn. Theor Appl Genet 85:961-968

Highton R, Webster TP (1976) Geographic protein variation and divergence in populations of the salamander, Plethodon cinereus. Evolution 30:33-45

Hirschfeld BM, Dhar AK, Rask K, Alcivar-Warren A (1999) Genetic diversity in the eastern oyster (Crassostrea virginica) from Massachusetts using the RAPD technique. J Shellfish Res 18:121-125

Holm S (1979) A simple sequentially rejective multiple test procedure. Scan J Stat 6:65-70

Huff DR, Peakall R, Smouse PE (1993) RAPD variation within and among natural populations of out crossing buffalo grass (Buchloe dactyloides [Nutt.] Engelm.). Theor Appl Genet 86:927-934

Kazan K, Manners JM, Cameron DF (1993) Genetic relationship and variation in the Stylosanthes guianensis species complex assessed by random amplified polymorphic DNA. Genome 36:43-49

Kimberling DN, Ferreira AR, Shuster SM, Keim P (1996) RAPD marker estimation of genetic structure among iso- 
lated northern leopard frog populations in the southwestern USA. Mol Ecol 5:521-529

Koh TL, Khoo G, Fan LQ, Phang VPE (1999) Genetic diversity among wild forms and cultivated varieties of Discus (Symphysodon spp.) as revealed by random amplified polymorphic DNA (RAPD) fingerprinting. Aquaculture 173:485-497

Kotoulas G, Bonhomme F, Borsa P (1995) Genetic structure of the common sole Solea vulgaris at different geographical scales. Mar Biol 122:361-375

Larcson JM, Riccardi VM, Calhoun SW, Morizot DC (1989) Genetic differentiation of bicolor damselfish (Eupomacentrus partitus) populations in the Florida Keys. Mar Biol 103:445-451

Lauder GV, Liem KF (1983) The evolution and interrelationships of the actinopterygian fishes. Bull Mus Comp Zool 150:95-197

Liu ZJ, Li P, Argue BJ, Dunham RA (1999) Random amplified polymorphic DNA markers: usefulness for gene mapping and analysis of genetic variation of catfish. Aquaculture 174:59-68

Lynch M, Milligan BG (1994) Analysis of population genetic structure with RAPD markers. Mol Ecol 3:91-99

Mamouris Z, Stamatis C, Bani M, Triantaphyllidis, C (1999) Taxonomic relationships between four species of the Mullidae family revealed by three genetic methods: allozymes, random amplified polymorphic DNA and mitochondrial DNA. J Fish Biol 55:572-587

Mantel N (1967) The detection of disease clustering and a generalised regression approach. Cancer Res 27:209-220

Martinez I, Pastene LA (1999) RAPD-typing of central and eastern north Atlantic and western north Pacific minke whales, Balaenoptera acutorostrata. ICES J Mar Sci 56:640-651

McCave IN, Caston VND, Fannin NGT (1977) The Quaternary of the North Sea. In: Shotton FW (ed) British Quaternary studies. Oxford University Press, Oxford, p 187-204

Naish KA, Warren M, Bardakci F, Skibinski DOF, Carvalho GR, Mair GC (1995) Multilocus DNA fingerprinting and RAPD reveal similar genetic relationships between strains of Oreochromis niloticus (Pisces: Cichlidae). Mol Ecol 4: 271-274

Nei M (1987) Molecular evolutionary genetics. Columbia University Press, New York

Nesbo CL, Fossheim T, Vollestad LA, Jakobsen KS (1999) Genetic divergence and phylogeographic relationships among European perch (Perca fluviatilis) populations reflect glacial refugia and postglacial colonization. Mol Ecol 8:1387-1404

Nusser JA, Goto RM, Ledig DB, Fleischer RC, Miller MM (1996) RAPD analysis reveals low genetic variability in the endangered light-footed clapper rail. Mol Ecol 5:463-472

Park LK, Moran P (1995) Developments in molecular genetic techniques in fisheries. In: Carvalho GR, Pitcher TJ (eds) Molecular genetics in fisheries. Chapman \& Hall, London, p 1-28

Peakall R, Smouse PE, Huff DR (1995) Evolutionary implications of allozyme and RAPD variation in diploid populations of dioecious buffalo grass, Buchloe dactyloides. Mol Ecol 4:135-147

Prager EM, Wilson AC (1976) Congruency of phylogenies derived from different proteins. J Mol Evol 9:45-57

Puterka GJ, Black WC, Steiner WM, Burton RL (1993) Genetic variation and phylogenetic relationships among worldwide collections of the Russian wheat aphid, Diuraphis noxia (Mordvilko), inferred from allozyme and PCR-RAPD markers. Heredity 70:604-618

Rice WR (1989) Analysing tables of statistical tests. Evolution $43: 223-225$
Rijnsdorp AD, Van Beek FA, Flatman S, Millner RM, Riley JD, Giret M, De Clerck R (1992) Recruitment of sole stocks Solea solea L. in the Northeast Atlantic. Neth J Sea Res 29: 173-192

Rohlf FJ (1993) NTSYS-pc Numerical taxonomy and multivariate analysis system, Version 1.80, Applied Biostatistics, Setauket, NY

Rossetto M, Weaver PK, Dixon KW (1995) Use of RAPD analysis in devising conservation strategies for the rare and endangered Grevillea scapigera (Proteaceae). Mol Ecol 4: 321-329

Schaal BA, O'Kane-Jr SL, Rogstad SH (1991) DNA variation in plant populations. Trends Ecol Evol 6:329-336

Singh RS, Rhomberg LR (1987) A comprehensive study of genic variation in natural populations of Drosophila melanogaster. II. Estimates of heterozygosity and patterns of geographic variation. Genetics 117:255-271

Slatkin M (1985) Gene flow in natural populations. Annu Rev Ecol Syst 16:393-430

Slatkin M (1993) Isolation by distance in equilibrium and nonequilibrium populations. Evolution 47:264-279

Smith PJ, Benson PG, McVeagh SM (1997) A comparison of three genetic methods used for stock discrimination of orange roughy, Hoplostethus atlanticus: allozymes, mitochondrial DNA, and random amplified polymorphic DNA. Fish Bull 95:800-811

Sneath PHA, Sokal RR (1973) Numerical taxonomy. Freeman, San Francisco

Stewart CN, Excoffier L (1996) Assessing population genetic structure and variability with RAPD data: Application to Vaccinium macrocarpon (American cranberry). J Evol Biol 9:153-171

Swofford DL, Selander RB (1989) Biosys-2: A computer program for the analysis of allelic variation in population genetics and biochemical systematics. Illinois Natural History Service. Champaign, Illinois

Symonds DJ, Rogers SI (1995) The influence of spawning and nursery grounds on the distribution of sole, Solea solea (L.) in the Irish Sea, Bristol Channel and adjacent areas. J Exp Mar Biol Ecol 190:243-261

Thorpe JP (1982) The molecular clock hypothesis: biochemical evolution, genetic differentiation and systematics. Annu Rev Ecol Syst 13:139-168

Thorpe JP, Sole-Cava AM (1994) The use of allozyme electrophoresis in invertebrate systematics. Zool Scr 23:3-18

Thorpe JP, Sole-Cava AM, Watts PC (2000) Exploited marine invertebrates: genetics and fisheries. Hydrobiologia 420: 165-184

Todd CD, Walker AM, Wolff $K$, Northcott SJ, Walker AF, Ritchie MG, Hoskins R, Abbott RJ, Hazon N (1997) Genetic differentiation of populations of the copepod ses louse Lepeophtherius salmonis (Kroyer) ectoparasitic on wild and farmed salmonids around the coasts of Scotland: Evidence from RAPD markers. J Exp Mar Biol Ecol 210: 251-274

Utter FM (1995) Perspective of molecular genetics and fisheries into the 21st century. In: Carvalho GR, Pitcher TJ (eds) Molecular genetics in fisheries. Chapman \& Hall, London, p 105-109

Utter FM, Aebersold P, Winans G (1987) Interpreting genetic variation detected by electrophoresis. In: Ryman N, Utter FM (eds) Population genetics and fishery management. University of Washington Press, Seattle, p 21-46

Vaisanen RA, Lehvasliho H (1984) Absence of genic polymorphism in a northern population of the house sparrow, Passer domesticus. Hereditas 100:161-162

Wang G, Whittam TS, Berg CM, Berg DE (1993) RAPD 
(arbitrary primer) PCR is more sensitive than multilocus allozyme electrophoresis for distinguishing related bacterial strains. Nucleic Acids Res 21:5930-5933

Ward RD (1989) Molecular population genetics of marine organisms. In: Ryland JS, Tyler PA (eds) Reproduction, genetics and distribution of marine organisms. Olsen \& Olsen, Fredensborg, p 235-249

Ward RD, Grewe PM (1995) Appraisal of molecular genetic techniques in fisheries. In: Carvalho GR, Pitcher TJ (eds) Molecular genetics in fisheries. Chapman \& Hall, London, p 29-54

Weir BS, Cockerham CC (1984) Estimating F-statistics for the analysis of population structure. Evolution 38:1358-1370

Welsh J, McClelland M (1990) Fingerprinting genomes using PCR with arbitrary primers. Nucleic Acids Res 18: $7213-7218$

Editorial responsibility: Otto Kinne (Editor),

Oldendorf/Luhe, Germany
Welsh J, McClelland M (1991) Genomic fingerprinting using arbitrarily primed PCR and a matrix of pair wise combinations of primers. Nucleic Acids Res 19:5275-5279

Wheeler A (1969) The fishes of the British Isles and NorthWest Europe. McMillan, London

Williams DJ, Kazianis S, Walter RB (1998) Use of random amplified polymorphic DNA (RAPD) for identification of largemouth bass subspecies and their intergrades. Trans Am Fish Soc 127:825-832

Williams JGK, Kubelik AR, Livak KJ, Rafalski JA, Tingrey SV (1990) DNA polymorphisms amplified by arbitrary primers are useful as genetic markers. Nucleic Acids Res 18: 6531-6535

Wright S (1978) Variability within and among natural populations. Evolution and the genetics of populations, Vol 4 . University of Chicago Press, Chicago

Submitted: August 15, 2001; Accepted: June 7, 2002

Proofs received from author(s): January 6, 2003 